\title{
Realidade Aumentada: Visualização Tridimensional e Interatividadena Documentação do Património Arquitetônico
}

\author{
Augmented Reality: Three-Dimensional Visualization and Interaction in Architectural Heritage Documentation
}

\author{
Carla Maria Furuno Rimkus \\ Universidade Federal de Sergipe, Brasil \\ carlarimkus@gmail.com
}

\author{
Fernando de Medeiros Galvão \\ Universidade Federal de Sergipe, Brasil \\ fernandomgalvao@gmail.com
}

\begin{abstract}
This paper presents preliminary results from an outgoing research about the Scholarship Program on Technological Development and Innovation (PIBITI), in which we focused the potentiality of Augmented Reality (AR) application on documentation of the architectural heritage according to the UNESCO principles. We took as the object of study of the "Quarteirão dos Trapiches", one of the historic buildings in Laranjeiras, Sergipe, Brazil, and we reconstructed it digitally with Augmented Reality and subsequently generate a QR Code information associated with this building, available on a website. In this context, this work highlight the potential application of architectural heritage documentation in AR, where discourse about the digital processing RA we use, and the association of this digital model with a history of the building. Finally, we present our conclusions on the use of RA in shareholders highlighting the advantages of three-dimensional visualization of architectural heritage, in an interactive, dynamic and low cost of deployment, pondering the importance of encouraging a policy of initiating development activities and technological innovation to digital architectural documentation.
\end{abstract}

Keywords: Augmented reality; Architectural heritage; Heritage education.

\section{Introdução}

Na América Latina, o Patrimônio Cultural foi menosprezadopor muito tempo, visto sob uma ótica eurocêntrica, ficando a margem dos estudos da história por muitas décadas.

Como bem observa Pellegrini, "A falta de estudos sistemáticos, a pequena divulgação e o preconceito estenderam-se até quase a metade do século passado quando surgiram projetos voltados ao turismo cultural e ao desenvolvimento sustentável em várias regiões do globo terrestre, desencadeando processos de reabilitação das áreas degradadas, sugerindo novos usos aos bens recuperados e a proteção e o manejo adequado do meio ambiente" (Pellegrini, 2006).

Laranjeiras, cidade histórica brasileira, situada em Sergipe, no Brasil é um exemplo que bem ilustra essa situação, onde a revitalização vem ocorrendo de forma superficial, privilegiando a "restauração de fachada" dos seus edifícios históricos, situação que vem fomentando a homogeneização do seu centro histórico, onde já se pode constatar uma impressão de conjunto forjada.

No Brasil, lamentavelmente observamos que os poderes públicos e a iniciativa privada têm investido em programas superficiais que criam simulacros de preservação ou cenários esvaziados de historicidade, a exemplo do Largo do Pelourinho(Bahia-Brasil) e de muitas outras cidades brasileiras, cujo resultado, não raro, configura-se como um pastiche, na busca do desenvolvimento do turismo, por vezes de um turismo indiscriminado e prejudicial, frequentemente dissociado das prerrogativas do turismo cultural.

Diante desse quadro, destacamos o grande desafio que consiste em promover a recuperação dos centros históricos dentro de uma perspectiva sustentável e de acordo com os princípios preservacionistas da UNESCO.

Nesse sentido, é imperativo convergir esforços integradores de estratégias de conservação, gestão e planejamento do patrimônio arquitetôniconos processos de desenvolvimento locais.

Diante desse quadro, torna-se urgente fundamentar uma metodologia adequada à recuperação do patrimônio arquitetônico de Laranjeiras,em vias de degradação, a partir de técnicas de revalorização econômica, social e estética e é nesse contexto que desenvolvemos este trabalho, enfocando como ponto central a avaliação de procedimentos metodológicos relativos à documentação do patrimônio arquitetônico com vistas à preservação e gestão desse patrimônio aplicando a tecnologia da RA.

Tomamos como objeto de estudo um conjunto de edifícios conhecido como Quarteirão dos Trapiches, que foi recentemente restaurado e adaptado para o funcionamento do campus Laranjeiras da Universidade Federal de Sergipe, Brasil. 


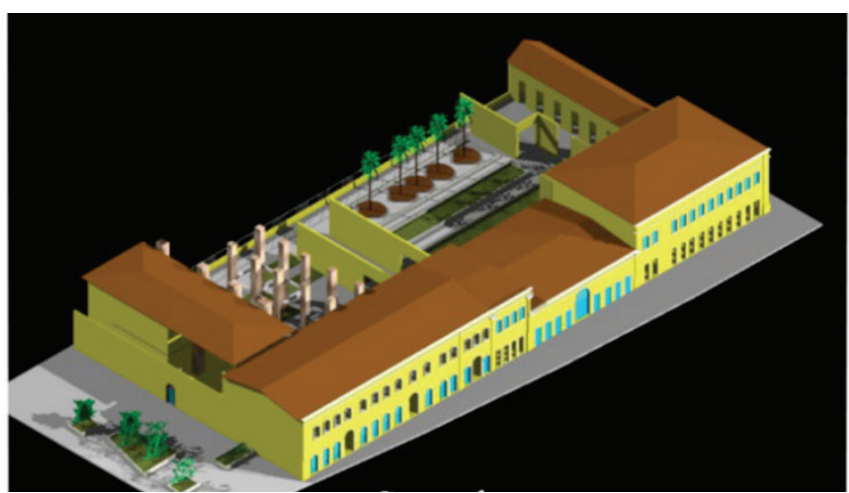

Figura 1: Quarteirão dos Trapiches. (Fonte: Barbara, Galdesson, Thacio NAU-UFS)

Nesse contexto, abordamos nesse artigo o desenvolvimento da documentação arquitetônica do Quarteirão dos Trapiches, enfocando como ponto central a avaliação de procedimentos metodológicos relativos àessa documentação com vistas à preservação e gestão desse patrimônio.

Nessa perspectiva de abordagem vamos utilizar a tecnologia da RA, especificando o sistema de visão por vídeobaseado em monitor para organizar a documentação técnica do patrimônio arquitetônico.

\section{Reabilitação e Preservação do Patrimônio Arquitetônico de Laranjeiras}

O conjunto urbanístico e arquitetônico de Laranjeiras congrega um passado histórico do período colonial e, em certos momentos, do neoclassicismo e do ecletismo brasileiros, narra a história de uma cidadeque detém traços visíveis da riqueza cultural brasileira e que a classificam como um patrimônio arquitetônico dos mais importantes no Brasil, tombado pelo IPHAN, mas entretanto, a despeito da sua importância verificamos a falta de documentação técnica da maior parte desse patrimônio, particularmente importante nesse momento em que a cidade de Laranjeiras passa por um processo de reabilitação e conservação do seu patrimônio arquitetônico, coordenado pelo programa Monumenta, pelo Instituto do Patrimônio Histórico e Artístico Nacional (IPHAN) e pela Prefeitura Municipal de Laranjeiras.

A vocação turística da cidade de Laranjeiras, tradicionalmente descrita como um "museu a céu aberto" já está consolidada pela presença constante de visitantes que chegam a cidade em busca de conhecimento sobre o seu patrimônio arquitetônico, vestígio de passado em que a cidade possuía o status do maior centro comercial do estado de Sergipe, devido ao escoamento da produção açucareira através de seu porto.

A cidade que vivenciou um período de glória, impulsionado pelo progresso econômico, também vivenciou um longo período de estagnação econômica, sendo que só recentemente vem experimentando um processo de revitalização e restauro. A maior parte dos seus edifícios de interesse histórico da cidade está relativamente bem preservada, mas um significativo número desses edifícios encontra-se em avançado estado de deterioração provocada pela ação do tempo.

Nesse contexto, um dos desafios das políticas de reabilitação dos sítios históricos é torná-los novamente produtores de alternativas econômicas e nesse sentido, indo de encontro às políticas de preservação sustentável do patrimônio arquitetônico e no âmbito do convênio estabelecido entre a Prefeitura Municipal de Laranjeiras e a Universidade Federal de Sergipe, esta pesquisa se desenvolve enfocando a tecnologia da Realidade Aumentada aplicada à reconstrução virtual do Quarteirão dos Trapiches em suas diferentes fases evolutivas, com o propósito de configurar um acervo técnico que será codificado através de um QR Code, onde serão disponibilizadas informaçõesem um websiteem ambientes virtuais 3D interativo, onde o usuário possa imergir, navegar e interagir em um ambiente sintético tridimensional, de forma natural e intuitiva, utilizando canais multissensoriais. Esse conteúdo será disponibilizado para o público em geral e para um público especializado composto por arquitetos, arqueólogos, museólogos e profissionais de áreas afins.Laranjeiras, cidade onde se situa o nosso objeto de estudo, está localizada em Sergipe, no Brasil, e é uma das cidades históricas mais importantes no Brasil, com um rico patrimônio arquitetônico.

A cidade de Laranjeiras tem no turismo históricoum forte potencial econômico, capaz de gerar postos de trabalho, riquezas e promover uma melhor distribuição de renda e a inclusão social na cidade, que segundo o IBGE tem um índice de pobreza superior a $60 \%$.

Nesse quadro de conscientização do patrimônio arquitetônico, que concorre para o desenvolvimento do turismo cultural enquanto recurso econômico, as facilidades proporcionadas pelos meios e tecnologias digitais de processamento, transmissão e armazenamento de informações reduzem custos e aumentam a eficácia dos processos de criação, troca e divulgação do patrimônio arquitetônico, mas apesar das evidentes vantagens, não se pode descuidar da exigência de rigor no conhecimento do passado, o que implica em novas formas de gestão desses valores e nesse aspecto é imperativo que se formule diretrizes para utilização de tecnologias digitais de um modo geral, que pela sua própria natureza são vulneráveis devido ao rápido obsoletismo de hardware e de software.

\section{Visualização e Interação em Ambiente Computacional Permeiam o Processo Histórico}

Ronald Azuma define a RA como um ambiente que envolve tanto realidade virtual como elementos do mundo real, criando um ambiente misto em tempo real. Trata-se de um sistema que: 
- combina elementos virtuais com o ambiente real;

- é interativo e tem processamento em tempo real;

- é concebido em três dimensões.

A Realidade Aumentada possibilita umavisualização tridimensional do edifício, propiciando a compreensão dos significados da história associada aos espaços, o que sem dúvida resulta numa melhor compreensão do edifício no contexto histórico, pois permite mostrar de maneira dinâmica as diferentes alterações dos edifícios históricos ao longo do tempo, bem como a sua interação no contexto urbano de forma dinâmica e com baixo custo.

Entretanto, apesardas evidentes vantagens da RA no contexto da documentação e gestão do patrimônio arquitetônico a utilização da tecnologia da RA ainda é tímida e na área da Arquitetura e Urbanismo tem caráter publicitário.

Dentre os seis diferentes sistemas de Realidade Aumentada optamos pelo sistema de visão por vídeo baseado em monitor.

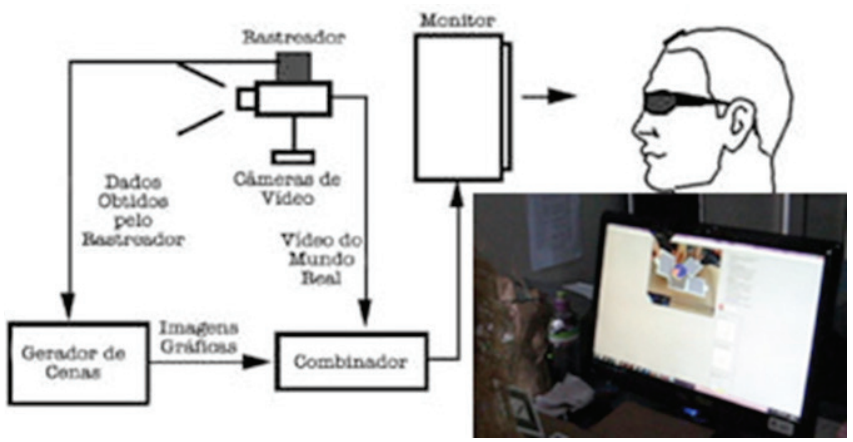

Figura 2: http://www.realidadeaumentada.com.br/aulas/Aula02. (Fonte: Ezequiel Zorzal)

Como se forma o objeto virtual?

1. Coloca-se um marcador em frente à câmera, que capta a imagem desse marcador e a transmite ao equipamento que fará a interpretação.(Figura 3)

2. A câmera visualiza esse marcador para o software que gerará o objeto virtual.

3. O software já estará programado para retornar o objeto virtual.

4. O dispositivo de saída (monitor de computador) exibe o objeto virtual em sobreposição ao real, como se ambos fossem uma coisa só.(Figura 4)

Neste trabalho, na sequência foi previsto o desenvolvimento de um website com ambientes virtuais 3D interativo, onde o usuáriopossaimergir, navegar e interagir em um ambiente sintético tridimensional, de forma natural e intuitiva, utilizando canais multissensoriais. Utilizando recurso Canvas da HTML 5 e o framework X3DOM comosuporte para a construção deste ambientesem a necessidade da instalação plug-ins no browser, integramos cenas X3D no código HTML o que permite a manipulação do conteúdo adicionando, removendo ou alterando dinamicamente os elementos DOM HTML. Obtivemos como resultado um ambiente virtual 3D onde o usuário pode navegar e interagir com os objetos do mundo tridimensional.

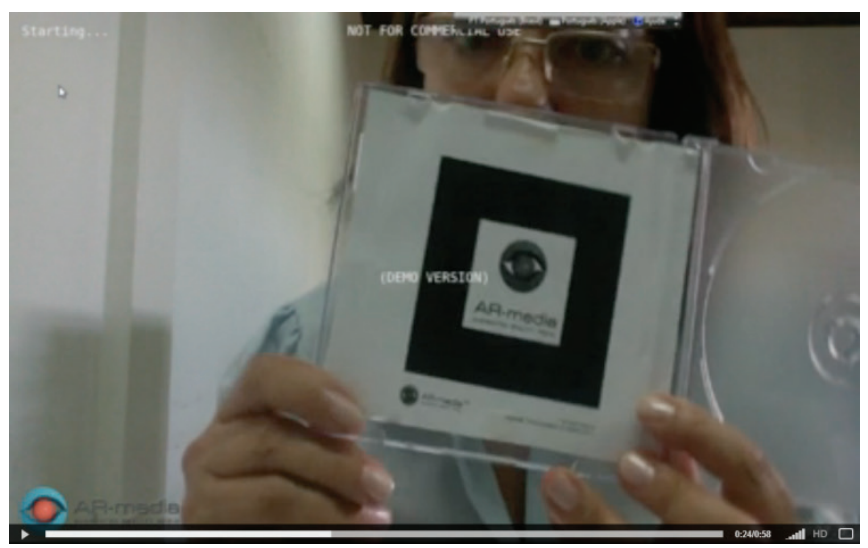

Figura 3: Marcador de Realidade Aumentada AR-media
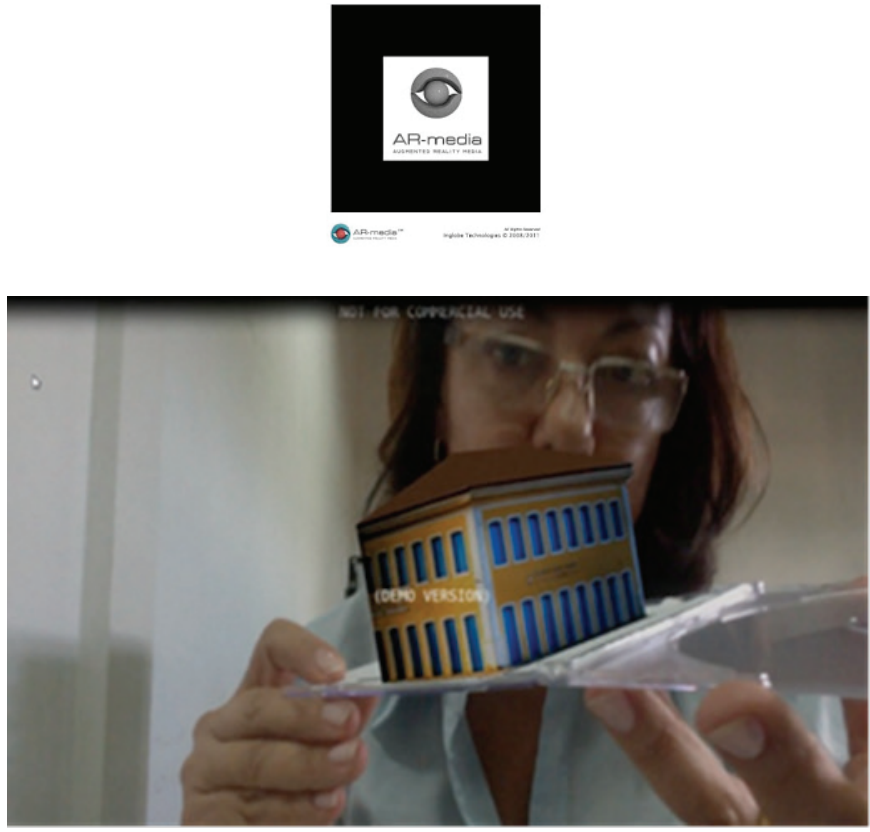

Figura 4: Quarteirão dos Trapiches em Realidade Aumentada. (Fonte: Carla Maria FurunoRimkus)

\section{Experiências de Realidade Aumentada Aplicada ao Patrimônio Arquitetônico}

Atualmente, no Brasil, alguns grupos de estudos acadêmicos desenvolvem pesquisas sobre visualização $3 D$ de patrimônio histórico-arquitetônico através de tecnologias apropriadas visando ampliar o acervo de experiências nessa área e, consequentemente, disponibilizar informações interativas que possibilitem uma melhor compreensão do espaço e dos objetos apresentados. 


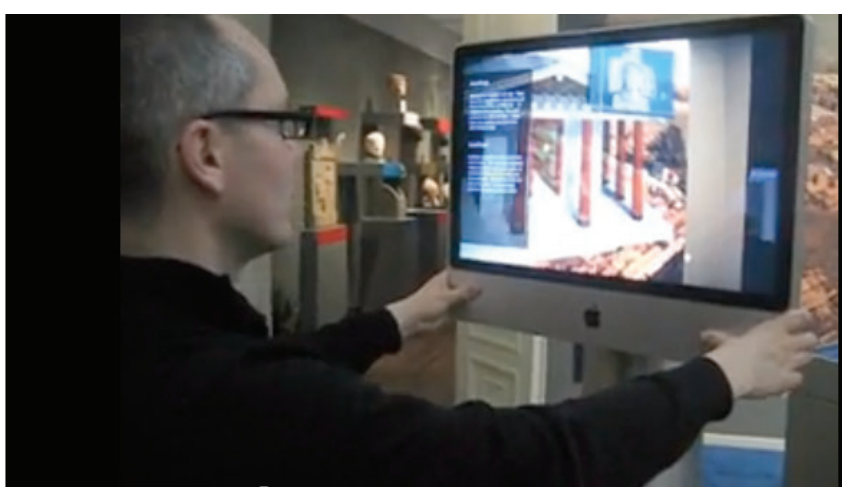

Figura 5: Tela Móvel - Museu AllardPiersonAmsterdã.(Fonte: http://www.tecmundo.com.br/novidade/5294-os-museus-ficaram-bemmais-divertidos-.htm)

Nesse contexto, a cidade de Pelotas, Rio Grande do Sul, Brasil, tem-se destacado no cenário nacional devido à geração de modelos tridimensionais digitais referenciados ao seu patrimônio e a parcerias com instituições de ensino superior que investem em pesquisas na área de realidade virtual. No cenário internacional, a Grécia tem investido na reconstrução de algumas de suas ruínas do seu sítio arqueológico como forma de melhorar o acervo das pesquisas sobre levantamento arqueológico e arquitetônico dos seus monumentos. É o caso, por exemplo, da aplicação de RA ao Templo de Olímpia.
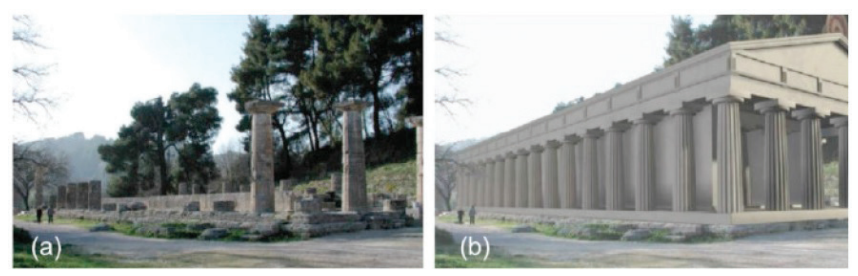

Figura 6: Templo de Olímpia: a) ruína em estado atual, e b) modelo virtual em RA. (Fonte: VLAHAKIS, 2002, apud AMORIM, 2013)

De forma análoga, a cidade de Laranjeiras apresenta-se como importante tema de interesse científico e didático e, especificamente, o Quarteirão dos Trapiches, objeto desse estudo, tem-se demonstrado como um caso relevante de uma primeira experiência da aplicação da RA ao patrimônio local. Sendo esse um resultado bem-sucedido, outros prédios históricos serão modelados virtualmente e, após uma otimização dos seus modelos geométricos, serão disponibilizados em RA para uma apreciação plena em hipermídia.

\section{Considerações Finais}

A utilização das técnicas de Realidade Aumentada na área do patrimônio arquitetônico contribui de forma única na compreensão do espaço e da história associada à edificação de forma dinâmica e com baixo custo de implantação, entretanto há que se observar que a utilização das tecnologias digitais de forma geral devem ser sistematizadas para aplicação no patrimônio arquitetônico para definir uma estrutura padronizada de metadados que observem procedimentos e estratégias de gestão arquivística de documentos segundo os princípios da Carta de Preservação Arquivística Digital da UNESCO, sob pena de não se garantir a acessibilidade a esta documentação em formato digital ao longo do tempo, tendo em vista o rápido obsoletismo que caracteriza a área de tecnologias digitais em termos de software e hardware.

\section{Referências}

AMORIM, Arivaldo Leão; SANTOS, Taís de Souza; HABEYCHE, Sthefania Campos. Virtual Heritage ou Tecnologias Digitais para a Visualização do Patrimônio Arquitetônico. Disponível em: http://dc361.4shared.com/doc/Rpqipfx2/preview.html. Acesso em 30/09/2013.

AZUMA, R. T. A Survey of Augmented Reality,Presence: Teleoperators and Virtual Environments,v.6, n.4, p.355-385, 1997.

CARAMELO, Frank. Imergindo na Arquitetura com Realidade Aumentada.In: $\quad$ http://www.portaldoarquiteto.com/blog/frankcaramelo/4326-imergindo-na-arquitetura-com-a-realidadeaumentada. Acesso em 01/09/2013.

FREIRE, Gerson José de Mattos. Escola de Arquitetura da UFMG, Brasil: Realidade Aumentada no Ambiente do Patrimônio Histórico.http://disegnarecon.unibo.it/article/viewFile/3320/2697. Acesso em 23/05/2013.

COULOMB, René. Modelos de gestiónen centros históricos de América Latina y Caribe. En busca de laintegridad, lagobernabilidad democrática y lasostenibilidad. In: CARRIÓN, Fernando. La ciudad construída: urbanismo en América. Quito: FacultadLatinoamericana de CienciasSociales-Junta de Andalucia/Rispergraf, 2001.

GARGNANI, Simone; MANFERDINI, Anna Maria. In SIGraDi 2011.Virtual and augmented reality applications for Cultural Heritage.p.556-559.

LÉVY, P. As tecnologias da Inteligência; o futuro do pensamento na era da informática. Rio de Janeiro: Ed.34, 1993.

LÉVY, P. O que é Virtual. São Paulo: Ed.34, 1996.

PELEGRINI, Sandra C. A. Cultura e patrimônio histórico. Estratégias de preservação e reabilitação da paisagem urbana. Latinoamérica. Revista de EstudiosLatinoamericanos. México, Universidad Nacional de México, n.38, 2004.

PROMOTER DIGITAL CULTURE .Augmented reality: EnrichingCulture; Acesso em 01/09/2013

SILVA, Adriane. Otimização de Modelos digitais para Visualizaçãode Patrimônio Histórico-Arquitetônico em RealidadeAumentada. In: SIGraDi 2012, p.423-425

UNESCO, CONARQ. Carta para a preservação do patrimônioarquivístico digital.. Disponível em http://www.conarq.arquivonacional.gov.br/ media/carta.pdf. Acesso em 13/08/2013.

ZORZAL,Ezequiel. Disponível em Aula2.http://www.realidadeaumentada .com. br/aulas/Aula02_-_Introducao_RA_Aplicacoes.pdf. Acesso em $23 / 05 / 2013$

ZUFFO, Marcelo Knorick; LOPES, Roseli de Deus. Ambientes de Realidade Virtual e Realidade Aumentada na Preservação do Patrimônio Histórico. Laboratório de Sistemas Integráveis (LSI) Departamento de Engenharia de Sistemas Eletrônicos Escola Politécnica da Universidade de São Paulo. In: Computação gráfica: pesquisas e projetos rumo à Educação Patrimonial Seminário - São Paulo. 